VCC may allow for a more accurate approximation of actual DD of inhaled therapies when targeting a calculated ID for critical care patients. Supported by PUMS and Discovery Laboratories, Inc.

\section{FACULTY AND STUDENT PERCEPTIONS OF EVALUATION IN A GULF MEDICAL SCHOOL}

doi:10.1136/archdischild-2012-302724.1006

'EH Aburawi, ${ }^{2}$ S Shaban, ${ }^{2} \mathrm{M}$ McLean. 'United Arab Emirates University, FMHS; ${ }^{2}$ Medical Education, United Arab Emirates University, FMHS, Al Ain, United Arab Emirates

Background Student evaluation of faculty members' ability to provide quality learning experiences is required for both formative and summative purposes. The aim was to explore the perceptions of medical students and faculty towards and teaching evaluations.

Methods A 21-item questionnaire, adapted from the Schmelkin et al. (1997) inventory, evaluated learner and faculty perceptions of purpose, etiquette, confidentiality, outcome and attitude towards evaluation. A 5-point Likert-scale was used ( $1=$ strongly disagree to $5=$ strongly agree). Mean \pm SD of the scale were compared using the Mann-Whitney test.

Results Fifty-two (54\%) of faculty members and 80 (23\%) of students completed the questionnaire. While both faculty and students strongly agreeing that there was sufficient security in terms of confidentiality in the online evaluation (faculty $=3.67 \pm 1.0 \mathrm{vs}$. students $=3.59 \pm 1.2$ ), students, however, believed instructors could recognize individual student comments $(3.23 \pm 1.0$ vs. $2.35 \pm 1.1)$, $(p<0.0001)$. Students strongly agreed $(3.56 \pm 0.7)$ that culture allowed objective evaluation of teachers, while faculty were less convinced $(2.83 \pm 1.1),(p<0.0001)$. Faculty believed more strongly that they made changes to their teaching in response to student evaluation (3.91 \pm 1.0 vs. $3.40 \pm 0.9, p<0.0001$ ).

Conclusions While there was general agreement on the value of teacher evaluation, there were differences between faculty and students in terms of the confidentiality, what teachers did with their evaluation and whether evaluation led to improved practice. Educating teachers and learners of the purpose of evaluation as a transparent process for quality improvement is an imperative.

\section{USING STATECHARTS AND THE MODEL-VIEW-CONTROLLER ARCHITECTURE TO DEVELOP A NOVEL ON-LINE FULLY INTERACTIVE SIPAP DEVICE FOR TRAINING AND COMPETENCY ASSESSMENT}

doi:10.1136/archdischild-2012-302724.1007

C Bosman. Department of Neonatology, Nottingham University Hospitals NHS Trust, Nottingham, UK

Performance-based training and competency assessment of junior medical and nursing staff in the use of medical devices such as ventilators places significant demands on instructor time. The development of hi-fidelity on-line simulations of medical devices and clinical situations presents a new way for doctors and nurses to learn and be assessed.

State chart theory was developed to categorise and diagram highly complex flight systems in aviation. This design paradigm combined with the software engineering architecture of the modelview-controller allows for the creation of infinitesimally complex systems.

Such methodology was used to recreate the exact workings of the SiPAP infant flow driver (Carefusion, CA), which has been used to train and assess both medical and nursing staff in our unit, in a fully educationally 'safe' environment without an instructor.

The entire state chart for the device will be demonstrated as well as the working on-line simulator in an ePoster format.

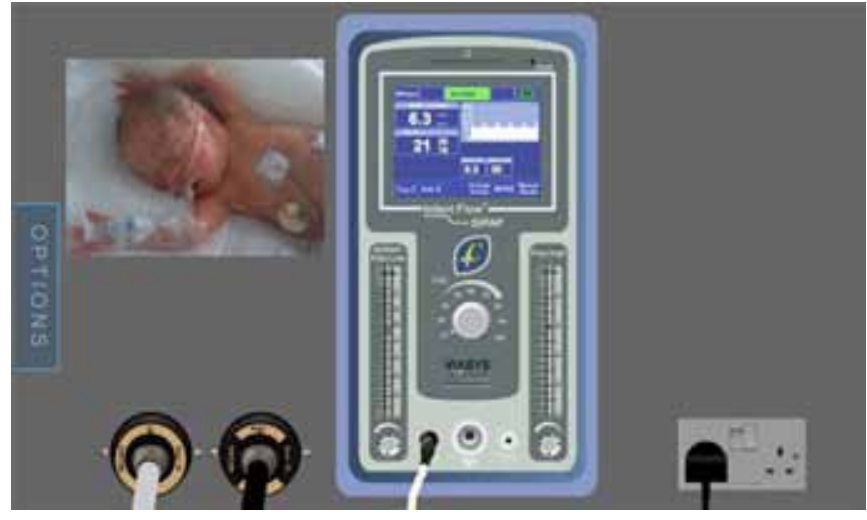

Abstract 1007 Figure 1 Simulator

\section{OPPORTUNITY COSTS IN PAEDIATRICS: THE SPECIALIST REGISTRAR EXPERIENCE}

doi:10.1136/archdischild-2012-302724.1008

N Kandamany, MB O’Neill. Mayo General Hospital, Castlebar, Ireland

Background and Aims Recently, the pursuit of a career in Paediatrics has gradually suffered a decline. This observation prompted an evaluation of Specialist Paediatric Registrars' (SPR) perceptions of their training programme and its impact on them.

Methods Utilising an online questionnaire, all trainees in 2011 were surveyed through the RCPI database. Data sought included demographics, perceptions of career progression, work effectiveness, social impact of hospital-based training and potential career costs in paediatrics. Likert scoring (cuing at 1 not at all, cuing at 6 extremely), binary questions (scored as yes, no or unsure) and narrative options were used.

Results Fifty-three (71\%) responses were obtained, with representation from each year of training. The mean Likert score was 3.8 for career progression, 4.6 for functional efficiency at work and 3.3 for attainment of a work-life balance. The value of log-books and endof-year assessments showed positive skewing (Likert 5/6) in $8(15 \%)$ and $7(13 \%)$ respectively. Narrative responses indicated the most challenging aspect of training was time for academic pursuits $28(53 \%)$. Other concerns included relocation for $8(15 \%)$ and career uncertainty for $7(13 \%)$. SPRs cited exercise for $29(43.4 \%)$ and discussion for 19 (35.8\%) as coping strategies. Thirty nine $(73.6 \%)$ SPR's incurred personal costs in training with compromised family cited by half. Mean Likert score for cost acceptability was 2.8 and perceived preparedness for consultancy, post-training, scored 3.7.

Conclusion SPRs feel there is a need for change within their scheme and that paediatric training incurs significant opportunity costs, with ultimate career uncertainty.

\section{CLINICAL UNCERTAINTY AND THE PAEDIATRIC TRAINEE}

doi:10.1136/archdischild-2012-302724.1009

'AM Deasy, 'M Elbadry, ${ }^{1} \mathrm{~A}$ Loftus, ${ }^{2} \mathrm{AJ}$ Nicholson, 'MB 0'Neill. 'Mayo General Hospital, Castlebar; ${ }^{2}$ Childrens University Hospital, Dublin, Ireland

Background and Aims Uncertainty is at the heart of clinical practice and is encountered by trainees at all levels. This study explored paediatrics trainees' perceptions of uncertainty.

Methods Basic specialist trainees in Paediatrics were surveyed while attending a training day. The survey explored 4 aspects related to uncertainty which included factors in decision making, personal 